\title{
Mixité, trajectoires professionnelles et division sexuée du travail dans les équipes infirmières
}

\author{
Séverine Rey \\ Sociologue-anthropologue, professeure HES ordinaire, Haute École de santé Vaud, Haute École \\ spécialisée de Suisse occidentale \\ Haute École de santé Vaud, 21 avenue de Beaumont, CH-1011 Lausanne, Suisse \\ severine.rey@hesav.ch
}

Les soins infirmiers sont une profession très féminisée qui inclut pourtant une certaine proportion d'hommes. Que signifie cette mixité assez réduite et quels effets a-t-elle sur le fonctionnement des équipes de travail et sur les trajectoires professionnelles?

Mots clés : division sexuée du travail ; genre ; mixité ; ségrégation ; soin infirmier

Les études sur le genre mettent en avant différents mécanismes à l'œuvre dans le cadre du travail et qui ont un impact tant sur la composition des professions que sur l'organisation même de l'activité professionnelle. Très largement féminisés, les soins infirmiers présentent un cas emblématique : après avoir explicité le lien entre soins et femmes, nous montrerons, d'une part, comment des hommes s'insèrent dans cette profession et, d'autre part, ce que cela produit au quotidien.

\section{Professions et division sexuée du travail}

Les soins infirmiers sont une profession très féminisée : selon les données publiées par la Direction de la recherche, des études, de l'évaluation et des statistiques (Dress) relatives à la démographie des professions de santé, 700988 infirmières et infirmiers étaient actifs en France en 2018, parmi lesquels $86,6 \%$ de femmes [1]. S'il n'y a pas lieu ici de revenir sur l'histoire de la profession et son évolution, on peut relever qu'une telle proportion n'est pas nouvelle (et n'évolue guère) ni spécifique à la France : elle est due à l'association traditionnelle des femmes au soin et à l'idée de vocation, ainsi qu'à leur exclusion de la médecine [2,3]. Comment comprendre cette situation ? L'un des apports phares des études sur le genre est l'analyse de la division sexuée du travail qui met en avant que les femmes ont été (et sont encore) assignées en priorité à l'espace domestique et reproductif, et les hommes à l'espace public et productif. Si l'on peut constater des variations dans le temps et dans l'espace quant aux modalités de la division (une tâche typiquement féminine dans une société peut être réservée aux hommes dans une autre), ces principes organisateurs sont partout les mêmes : "Le principe de séparation (il y a des travaux d'hommes et des travaux de femmes) et le principe hiérarchique (un travail d'homme "vaut" plus qu'un travail de femme) " [4]. Certains secteurs sont fortement féminisés (santé, éducation, social, etc.) et d'autres très masculinisés (industrie, construction, etc.) - la répartition entre les deux renvoyant aux rôles et aux valeurs généralement associés au féminin/aux femmes et au masculin/aux hommes. C'est sur cette base que se dessinent les choix de profession qui restent encore aujourd'hui fortement attachés au sexe, autrement dit aux représentations sociales liées à la division sexuée du travail. 


\section{Le choix de la profession infirmière pour les hommes}

Comment et pourquoi les hommes entrent-ils dans ce secteur féminisé ? Dans une recherche que nous avons menée sur le vécu de la formation en Suisse romande par des personnes qui ont choisi un domaine où leur sexe est sous-représenté ${ }^{1}$ [5], nous avons constaté que la plupart des hommes rencontrés avaient formulé leur choix de manière tardive et que le hasard avait parfois joué un rôle dans leur décision. En effet, au terme des études secondaires, ils ne savaient souvent pas ce qu'ils voulaient faire, et certains se sont laissé du temps pour voir et/ou ont accompli soit leur service militaire dans les troupes sanitaires (affiliation qu'ils n'avaient souvent pas choisie), soit leur service civil qui implique un temps de travail pour la collectivité, souvent dans des structures sociosanitaires ${ }^{2}$. Enfin, d'autres ont essuyé des échecs de formation universitaire et ont alors décidé de $s^{\prime}$ orienter vers les soins infirmiers.

On le voit, l'association sociale des soins à la féminité perdure et rend difficile l'aspiration professionnelle des hommes dans ce secteur. Ceux qui le rejoignent font l'expérience des stéréotypes liés à leur choix et vivent souvent des formes de stigmatisation. En effet, faire un métier de femme met à l'épreuve leur masculinité [6] : l'imaginaire collectif accole diverses étiquettes à leur choix (féminisation, soupçon d'homosexualité) contre lesquelles il leur faut résister et élaborer des stratégies de trajectoire professionnelle, par exemple.

\section{Mixité, ségrégation et inégalités}

Ainsi, les soins infirmiers restent massivement un secteur féminisé, incluant toutefois une certaine mixité. Que faut-il entendre par "mixité" ? Ce terme renvoie à la proportion d'hommes et de femmes dans un domaine professionnel : on prend souvent le seuil de $30 \%$ du sexe sous-représenté comme critère pour traiter des situations où les sexes coexistent dans un secteur qui peut être marqué par une suprématie numérique tantôt féminine, tantôt masculine [7]. Quelles sont les conséquences de la mixité sur la répartition de la population active féminine et masculine sur le plan hiérarchique (une réalité qu'on analyse, en sociologie, sous l'expression "ségrégation verticale") et sur leur répartition dans différents secteurs économiques, branches d'activité et métiers ("ségrégation horizontale") ?

Diverses études montrent que dans la plupart des cas où des professions typiquement "masculines" ou "féminines" ont connu une certaine mixité, la division sexuée du travail, au lieu de disparaître, s'est déplacée [8]. La ségrégation horizontale s'établit à l'intérieur même de la profession, par le biais de spécialisations qui recréent une forme d'opposition entre les sexes : par exemple, les infirmiers s'orientent majoritairement dans les soins dits aigus (anesthésie, urgences, etc. [9]). C'est notamment le cas en France : d'après les statistiques de la Dress sur la démographie des professions de santé, si l'on étudie les effectifs infirmiers selon les spécialisations, on constate qu'en 2018 seul $1,4 \%$ d'hommes sont actifs dans la puériculture alors qu'ils sont $31,1 \%$ en anesthésie et $46 \%$ sont cadres infirmiers en psychiatrie.

Par ailleurs, la mobilité ascendante est plus fréquente pour les hommes qui ont choisi une profession "féminine" : selon l'expression de la sociologue C.L. Williams [10], ils bénéficient d'un " escalator de

\footnotetext{
${ }^{1}$ Recherche exploratoire, menée par entretiens semi-directifs. Dans le but de comparer le vécu de la formation (de niveau supérieur) des jeunes femmes et hommes, nous nous sommes intéressées aux deux domaines les moins mixtes de la Haute École spécialisée de Suisse occidentale : l'ingénierie ( 8 \% de femmes) et la santé $(14,4$ $\%$ d'hommes), dont les soins infirmiers. (Chiffres 2011, date du début de la recherche.)

${ }^{2}$ En Suisse, le service militaire est obligatoire pour les hommes et peut être remplacé, sur demande motivée, par un service civil d'une durée une fois et demie supérieure au service militaire.
} 
verre ", soit d'invisibles pressions et soutiens pour monter en grade, en miroir de ce que vivent les femmes qui se heurtent au "plafond de verre", des barrières inapparentes mais bien présentes qui empêchent leur promotion. Par ailleurs, les inégalités entre les sexes se marquent très tôt : dans notre étude sur les trajectoires d'insertion professionnelle des diplômés du supérieur, minoritaires selon le sexe ${ }^{3}[11]$, nous avons constaté qu'un an après l'obtention du diplôme, $79 \%$ des personnes du domaine de la santé ont un contrat à temps plein, mais que ce taux tombe à $48 \%$ cinq ans après. Or, cette baisse est surtout imputable aux femmes, puisque $70 \%$ des hommes ont un contrat à plein temps ${ }^{4}$. Le temps partiel comme modalité d'emploi contribue non seulement à ralentir les carrières féminines, mais aussi, plus généralement, à creuser les inégalités entre les sexes. Les hommes actifs dans les domaines féminisés exercent moins souvent une activité à temps plein que les hommes des domaines masculinisés, mais leur taux d'occupation est tout de même plus élevé que celui de leurs collègues féminines.

\section{Mixité et travail au quotidien}

Les infirmières et infirmiers rencontrés durant notre enquête ${ }^{5}$ [12] mettent tous en avant leur professionnalisme, leur formation et leur expérience : c'est ce qui prime dans leur travail et leur prise en charge des patients. Les différences qu'ils relèvent ne sont pas liées à leur appartenance sexuée, mais à leur individualité : "Le travail infirmier, que ce soit un homme ou une femme qui l'accomplisse, c'est la même chose, car on a le même rôle et on a été formé de la même manière ", nous a déclaré un infirmier participant.

La mixité présente un avantage, selon eux : elle permet de répondre à des demandes spécifiques " dans l'intérêt des patients ", comme être pris en charge uniquement par un homme ou par une femme, que ce soit pour des raisons de vécu traumatique ou de conviction religieuse par exemple. Ils jugent cette souplesse pratique, mais critiquent également de telles requêtes dans la mesure où elles restreignent leur rôle lorsque, par exemple, des patients " nous voient comme des femmes ", remarque une infirmière : "On se retrouve ainsi un peu plus souvent à faire le boulot maternel. "

Cependant, la mixité dans les équipes infirmières a des conséquences en termes de répartition du travail. On constate certaines délégations qui sont associées à l'appartenance sexuée des individus et non à leurs compétences professionnelles : aux hommes, la force physique (malgré l'existence de moyens mécaniques) et l'autorité, voire la gestion de la violence (malgré l'apprentissage de chacun et chacune en matière de résolution des conflits) ; aux femmes, la prise en charge de l'intimité qu'elle soit celle d'une patiente ou d'un patient. Cela est bien évidemment paradoxal quand on sait que ces mêmes gestes et activités sont également réalisés quand les équipes sont non mixtes. Ainsi, c'est souvent la mixité qui réintroduit un réflexe associé à une forme de naturalisation et de différenciation entre les sexes.

\footnotetext{
${ }^{3}$ L'étude comparait les trajectoires de minoritaires, soit des femmes dans les domaines masculinisés de l'ingénierie et de l'architecture, soit des hommes dans les secteurs féminisés de la santé et du travail social. Outre une analyse secondaire de données statistiques relative à la situation professionnelle et privée des diplômés des hautes écoles spécialisées suisses, nous avons mené des entretiens semi-directifs avec des minoritaires, mais aussi des dirigeants d'entreprise ou d'institution et des responsables de ressources humaines.

${ }^{4}$ C'est une caractéristique frappante en Suisse : en 2016, 58,8 \% des femmes actives (tous secteurs confondus) travaillaient à temps partiel, contre $17,1 \%$ des hommes [13].

${ }^{5}$ Recherche sur la répartition du travail dans des équipes mixtes d'infirmiers-infirmières (service des urgences notamment) et de manipulatrices-manipulateurs en électroradiologie dans un centre hospitalier universitaire de Suisse romande. Nous avons eu recours à l'observation ethnographique et à des entretiens semi-directifs.
} 


\section{Conclusion}

Comme les métiers des soins sont encore souvent, dans la représentation collective, associés aux femmes et à une forme de vocation (niant les compétences), l'augmentation de la proportion d'hommes exerçant pourrait, un peu paradoxalement, les revaloriser, voire les professionnaliser. Cela participerait à résoudre la question de la pénurie endémique de personnel dans ce secteur. Cependant, la mixité peut être un problème lorsqu'elle n'est pas anticipée, accompagnée et gérée en connaissance de cause, car elle renforce les inégalités déjà existantes dans le monde du travail. C'est pourquoi les instances de formation et les lieux de travail devraient veiller non seulement à "dégenrer" le soin (l'empathie et la sollicitude ne sont pas, par nature, féminines) et en valoriser les très nombreux aspects techniques, mais également à offrir des conditions de travail et des trajectoires professionnelles détachées de l'appartenance sexuée et des modèles discriminants de carrière qui lui sont associées.

\section{Points à retenir}

- Les spécialisations au sein de la profession infirmière reproduisent, en la déplaçant, la division sexuée du travail ("ségrégation horizontale").

- Les trajectoires professionnelles sont marquées par l'appartenance sexuée (les infirmiers bénéficient de l'“escalator de verre").

- La mixité dans les équipes s'accompagne de délégations de gestes et de prises en charge en fonction du sexe et non des compétences professionnelles.

- La mixité dans les équipes doit être anticipée et accompagnée pour ne pas renforcer les inégalités.

\section{Références}

[1] La démographie des professionnels de santé. Ministère des Solidarités et de la santé 2020. https://drees.solidarites-sante.gouv.fr/etudes-et-statistiques/open-data/professions-de-santeet-du-social/la-demographie-des-professionnels-de-sante/article/la-demographie-desprofessionnels-de-sante.

[2] Leroux-Hugon V. L'infirmière au début du xxe siècle : nouveau métier et tâches traditionnelles. Le Mouvement social 1987;140:55-68.

[3] Cohen Y. Du nursing au care : le genre d'une profession. Aporia: The Nursing Journal 2012;4(1):42-50.

[4] Kergoat D. Se battre, disent-elles... Paris: La Dispute; 2012. p. 214.

[5] Rey S, Battistini M. Chassez les stéréotypes, ils reviennent au galop! Choix atypiques de formation professionnelle et différenciation des groupes de sexe. Éducation et socialisation 2016;42. http://journals.openedition.org/ edso/1831.

[6] Buscatto $M$, Fusulier N. Présentation. Les "masculinités" à l'épreuve des métiers "féminins". Recherches sociologiques et anthropologiques 2013; 44(2):1-19.

[7] Fortino $S$. De la ségrégation sexuelle des postes à la mixité au travail : étude d'un processus. Sociologie du travail 1999;41(4):363-84.

[8] Fortino S. La mixité au travail. Paris: La Dispute; 2002.

[9] Snyder KA, Green Al. Revisiting the glass escalator: the case of gender segregation in a female dominated occupation. Social Problems 2008;55(2):271-99. 
[10] Williams CL. The glass escalator: hidden advantages for men in the "female" professions. Social Problems 1992;39(3):253-67.

[11] Fasel R, Kuehni M, Rey S. Impact du genre sur l'insertion des diplômés du supérieur dans quatre domaines d'activité en Suisse. Formation Emploi 2019;145:53-78

[12] Rey S, Battistini M, Pirinoli $C$. La mixité produit-elle des effets ? Organisation du travail et partage de la pratique quotidienne dans des équipes de professionnels de la santé. In: Anderfuhren $M$, Rodari S (dir.). Sans garantie de mixité. Genève: Éditions IES 2014;151-67.

[13] Dubach $\mathrm{P}$, Legler V, Morger M, Stutz H. Frauen und männer an schweizer hochschulen : indikatoren zur chancengleichheit in studium und wissenschaftlicher laufbahn. Bern: SBFl; 2017. 